Chimia 45 (1991) 53-54

(C) Schweiz. Chemiker-Verband; ISSN $0009-4293$

\section{Qualitativer Bodenschutz in der Schweiz - eine Standortbestimmung}

\author{
Heinz Häni*
}

Abstract. The present paper deals with the efforts made in Switzerland to treat the qualitative soil protection (monitoring and assessment of soil pollution mainly by heavy metals) on a scientific and legislative basis. In addition to a critical appreciation of guide levels for pollutants in soil, indications on important gaps in research are given (e.g. risk assessment in soils with exceeded guide levels or organic micropollutants).

\section{Einleitung}

Auf wissenschaftlicher Ebene beschäftigt man sich in der Schweiz seit ca. 20 Jahren mit Fragen des qualitativen Bodenschutzes. Es geht dabei um die Erfassung und Beurteilung von Bodenbelastungen durch Schadstoffe, die aus der Luft, vom Umgang mit umweltgefährdenden Stoffen oder von Abfällen stammen.

Die gesetzliche Regelung basiert auf dem Umweltschutzgesetz vom 7 . Oktober 1983 , dessen Zweckartikel neben dem Schutz von Menschen, Tieren und Pflanzen, ihren Lebensgemeinschaften und Lebensräumen gegen schädliche oder lästige Einwirkungen auch die Erhaltung der Fruchtbarkeit des Bodens beinhaltet. Die Beurteilung der Belastung des Bodens mit schädlichen und nicht oder nur schwer abbaubaren Stoffen erfolgt mittels Richtwerten, die in der Verordnung über Schadstoffe im Boden vom 9. Juni 1986 (VSBo) vorläufig für 11 Schwermetalle und Fluor festgelegt wurden [1].

Trotz der Tatsache, gewisse Erkenntnisse bereits auf Verordnungsstufe umgesetz zu haben und einer zunehmenden Bodenforschungstätigkeit (erwähnt sei u.a. das $\mathrm{Na}$ tionale Forschungsprogramm 'Boden'), scheint die breite Bevölkerung die Bedeutung des qualitativen Bodenschutzes nur zögernd wahrzunehmen. Dies mag damit zusammenhängen, dass eine Belastung, anders als bei Luft und Wasser, nicht so unmittelbar sichtbar ist. Schadstoffe werden entweder im Boden festgehalten (Schwermetalle) oder abgebaut (gewisse organische Verbindungen). Damit wird ihre Schadwirkung auf

\footnotetext{
*Korrespondenz: Dr. H. Häni

Eidgenössische Forschungsanstalt für

Agrikulturchemie und Umwelthygiene CH-3097 Liebefeld
}

Pflanzen und Bodenlebewesen sowie die Gefahr einer Auswaschung ins Grundwasser verzögert. In jedem Boden treten allerdings nach Überschreiten einer bestimmten Schwelle je nach Bodeneigenschaften früher oder später negative Effekte auf.

Nun wirkt sich die mangelnde Erkenntnis, dass einem Boden nicht alles und jedes zuzumuten ist, dass man ihn also nicht als Schadstoffdeponie missbrauchen darf, äusserst verhängnisvoll aus. Im Gegensatz zu Luft und Wasser lässt sich nämlich ein verschmutzter Boden kaum mehr reinigen. Man stösst hier an die Grenzen der technischen
Möglichkeiten, indem es praktisch kein Verfahren zur Reinigung eines Bodens ohne seine gleichzeitige Zerstörung gibt. Gerade deshalb ist es wichtig, ein Bewusstsein für den Boden zu schaffen, d.h. mit allem Nachdruck auf die Bedeutung des komplexen Ineinanderwirkens verschiedener unbelebter (Ton, Humus, Oxide) und belebter Komponenten (Bodenflora und -fauna) hinzuweisen.

\section{Erfassung von Bodenbelastungen mit Schwermetallen}

Aus dem Projekt 'Schwermetallgehalte in den Böden der Schweiz' des Nationalen Forschungsprogramms 'Boden' wurde ein Überblick der Gehalte der Schwermetalle Blei, Cadmium, Kupfer, Nickel und Zink gewonnen [2]. Ausgehend von 41 Standorten des Nationalen Bodenbeobachtungsnetzes fürSchadstoffe im Boden (NABO) wurde unter Berücksichtigung der lokal und regional vorherrschenden Standortfaktoren eine Messkampagne durchgeführt, welche sich über die meisten intensiv genutzten Gebiete unseres Landes (v.a. Mittelland) erstreckte. Als analytische Methode wurde zur Annäherung des Totalgehaltes der in der VSBo beschriebene $\mathrm{HNO}_{3}$-Auszug verwendet.

Ein Vergleich der Oberboden- $(0-20 \mathrm{~cm})$ mit den Unterbodengehalten $(60-80 \mathrm{~cm}$ oder tiefstmögliche Schicht aus dem gleichen Ausgangsmaterial wie der Oberboden) soll eine Abschätzung anthropogener Einflüsse erlauben. In der $T a b$. sind die gefundenen Ergebnisse zusammengestellt.

Die verschiedenen Standortfaktoren beeinflussen die einzelnen Schwermetalle im Oberboden in einem unterschiedlichen Ausmass.

So werden die Gehalte des Bleis durch Nutzung und Geologie kaum, durch einzelne Emissionen (Industriebetriebe, Kehrichtverbrennungsanlagen usw.) sowie diffuse Emissionsquellen (Agglomerationen, Verkehr) dagegen deutlich messbar beeinflusst.

Bei Cd und Zn spielen einzelne Emissionsquellen lokal eine wichtige Rolle. In den landwirtschaftlich genutzten Parzellen scheint bei diesen Elementen ein gewisser Eintrag stattzufinden, während in Waldpar-

Tabelle. Angenäherte Totalgehalte (Median und häufiger Bercich) ron Blei, Cadmium. Kupfer, Nickel und Zink in Oberboden- und Unterbodenproben der Schweiz. Zahlen in Klammern VSBo-Richtwerte in ppm.

\begin{tabular}{lllllll}
\hline \multirow{2}{*}{ Element } & \multicolumn{2}{l}{ Oberboden [ppm] } & \multicolumn{3}{l}{ Unterboden [ppm] } \\
\cline { 2 - 3 } & \multicolumn{2}{l}{ Median } & \multicolumn{2}{c}{ häufiger Bereich } & & \multicolumn{2}{c}{ Median } & häufiger Bereich \\
\hline Blei (50) & 23,8 & 13,5 & $-40,5$ & 12,1 & 7,8 & $-18,2$ \\
Cadmium (0,8) & 0,23 & 0,11 & $-0,45$ & 0,12 & 0,07 & $-0,23$ \\
Kupfer (50) & 23,3 & 9,0 & $-61,0$ & 13,8 & 7,8 & $-27,0$ \\
Nickel (50) & 23,5 & 12,5 & $-39,0$ & 24,8 & 14,5 & $-50,0$ \\
Zink (200) & 56,1 & 33,0 & $-94,0$ & 39,9 & 23,5 & $-60,0$ \\
\hline
\end{tabular}

zellen eher tiefere Gehalte gemessen wurden. Bezüglich Geologie und regionaler Unterschiede können keine sicheren Aussagen gemacht werden.

Die höchsten $\mathrm{Cu}-G e h a l t e$ weisen mit Abstand die Spezialkulturen (v.a. Rebbau) auf. Bei den Emissionsquellen wurden hauptsächlich in der Umgebung einzelner Industriebetriebe (Metallverarbeitung, Kehrichtverbrennungsanlagen) erhöhte Gehalte gemessen. In Landwirtschaftsböden scheint ein gewisser Cu-Eintrag stattzufinden, der jedoch selten Richtwertüberschreitungen zur Folge hat, während in Wald häufig sehr tiefe Werte gemessen wurden.

Ni weist als einziges Element mit höhe- 
ren Unterbodengehalten in der Westschweiz eindeutig regionale Tendenzen auf. Häufig werden in Oberboden geringere Gehalte als im Unterboden gefunden. Bezüglich Nutzung und verschiedener Emissionsquellen können keine Einflüsse nachgewiesen werden.

Auch wenn aus diesen Resultaten der Schluss gezogen werden darf, dass die Totalgehalte der untersuchten Schwermetalle in Landwirtschafts- und Waldböden mehrheitlich unterhalb der VSBo-Richtwerte liegen, muss doch darauf hingewiesen werden, dass aus anderen Messungen Flächen mit bedeutenden Schwermetallbelastungen bekannt sind. Allerdings können derartige Flächen in ihrer Gesamtheit bis heute noch nicht quantifiziert werden. Hier werden vermehrte Kenntnisse aus Untersuchungen kantonaler Bodenschutzfachstellen, die im Zusammenhang mit der VSBo geschaffen wurden, erwartet.

\section{Beurteilung von Schwermetallbela- stungen}

Die eben erwähnten angenäherten Totalgehalte lassen keine Beurteilung biologischer Wirkungen, die von den Schwermetallen im Boden ausgehen können, zu. Man hat dabei hauptsächlich an Wirkungen auf Pflanzen (Beeinträchtigung der Qualität wegen erhöhter Gehalte oder toxisch be dingte Ertragseinbussen) und Bodenmikroorganismen (Beeinflussung der Diversität, Vitalität sowie Abbauleistungen) zu denken.

Zahlreiche Untersuchungen haben gezeigt, dass neutrale Salzlösungen am besten geeignet sind, biologisch relevante Schwermetallkonzentrationen im Boden zu erfassen [3]. Aus diesem Grund sind in der VSBo Richtwerte sowohl für angenäherte Totalgehalte $\left(2 \mathrm{M} \mathrm{HNO}_{3}\right)$ als auch für lösliche Gehalte $\left.(0,1 \mathrm{M} \mathrm{NaNO})_{3}\right)$ enthalten.

Man kann sich natürlich die Frage stellen, ob Richtwerte für lösliche Gehalte nicht genügt hätten, da diese für die Abschätzung unmittelbar wirksamer Schwermetall-konzentrationen allein entscheidend sind. Ein solches Vorgehen hätte jedoch unerwünschte Konsequenzen, indem es möglich wäre, einen gut bindenden Boden ( $\mathrm{pH} \geq 7$, hoher Humus- und Tongehalt) total sehr stark zu belasten, was bei sich verändernden Bode- neigenschaften (pH-Absenkung, Humusabbau) zu einem Anstieg der löslichen Gehalte weit über den Richtwert hinaus führen würde.

\section{Kritik an Bodenrichtwerten}

Der VSBo kommt eindeutig präventiver Charakter zu, indem gemäss ihrem Wortlaut Richtwerte so festzulegen sind, 'dass nach dem Stand der Wissenschaft und der Erfahrungen Belastungen unterhalb dieser Werte die Fruchtbarkeit des Bodens auch langfristig nicht beeinträchtigen'. Beim Erreichen der Richtwerte oder deutlichen Ansteigen eines Schadstoffgehaltes treffen die Kantone nach Ermittlung der Schadstoffquellen Massnahmen in den Bereichen Luftreinhaltung, umweltgefährdende Stoffe und Abfälle.

Trotz diesem klaren Bekenntnis zur Vorsorge, das übrigens auch darin zum Ausdruck kommt, dass die Schweiz im internationalen Vergleich die tiefsten Bodenrichtwerte besitzt, wurde in jüngster Zeit zunehmend Kritik laut, die sich weniger auf die Höhe dieser Werte als vielmehr auf das Richtwertdenken im allgemeinen bezog. Mit diesem System lasse man eben doch zu, dass die Pflugschicht langsam, aber stetig mit Fremd- und Schadstoffen bis zum Erreichen der Richtwerte aufgefüllt werde. Stattdessen wird gefordert, landwirtschaftlich genutzten Böden nur noch so viel Fremd- und Schadstoffe zuzuführen, wie sie in unschädlicher Art und Weise wieder an Pflanzen, Wasser oder die Luft abgeben können [4]. Zur Erfassung solcher Fliessgleichgewichte müssen Stoffbilanzen, d.h. exakte Erhebungen über Ein- und Austräge von Stoffen erstellt werden.

Zu diesen zwei Ansätzen sei lediglich so viel gesagt, dass sie nicht als Gegensätze sondern vielmehr als parallel zu verfolgende Konzepte anzusehen sind. Längerfristig ist im Interesse eines wirksameren Bodenschutzes auf die Erreichung von Fliessgleichgewichten hin zu arbeiten, wobei die Lösung methodischer Probleme und die gesetzliche Umsetzung wesentlich schwieriger sein dürften als dies für die Festlegung von Richtwerten der Fall ist.

\section{Ausblick}

Aus dem präventiven Charakter der. VSBo wird verständlich, dass man sich bis heute nur wenig mit Massnahmen, die bodenseitig beim Überschreiten der Richtwerte zu ergreifen sind, beschäftigt hat. Da solche Belastungsflächen, wie bereits erwähnt, auch in der Schweiz vorkommen, sind dringend Handlungskonzepte mit einer breit abgestützten Risikobeurteilung zu entwickeln. Es gilt neben Fragen des Grundwasserschutzes und der Erfassung bodenmikrobiologischer Parameter zur Abschätzung einer Gefährdung der Bodenfruchtbarkeit abzuklären, ob und unter welchen Bedingungen eine bestimmte Nutzung noch verantwortbar ist.

Grosse Forschungsanstrengungen bedarf es auf dem Gebiet der organischen Schadstoffe (Verhalten im Boden; Abschätzung des Risikos solcher Stoffe für das Bodenökosystem). Daneben hat man zu überprüfen, wie weit die in letzter Zeit immer häufiger angebotenen Sanierungsverfahren ökonomisch und ökologisch vertretbar sind.

Schliesslich ist auch den Gefährdungen der Bodenfruchtbarkeit, die nicht oder nicht ausschliesslich auf den Eintrag von Schadstoffen zurückzuführen sind, vermehrt $\mathrm{Be}$ achtung zu schenken.

Mit der VSBo wurde ein erster wichtiger Schritt im qualitativen Bodenschutz getan. Wie die eben aufgeführten Lücken zeigen, bedarf es für seine notwendige Weiterentwicklung nicht unbedeutender zusätzlicher Anstrengungen

[1] Bundesant für Umwclt, Wald und Landschaft: Erläuterungen zur Verordnung vom 9. Juni 1986 über Schadstoffe in Boden (VSBo), Juni 1987.

[2] H. Vogel, A. Desaules, H. Häni, Schwermetallgehalte in den Böden der Schweiz, Bericht Nr. 40 des Nationalen Forschungsprogramms 'Boden', Dezember 1989

[3] S. K. Gupta, H. Häni, A. Rudaz, E. SantschiFuhrimann, A. Blatter, Methodik zur Bestimmung biologisch relevanter Schwermetallkonzentrationen im Boden und Ueberprüfung der Auswirkungen auf Testpflanzen sowie Mikroorganismen in belasteten Gebieten, Schriftenreihe der FAC, $\mathrm{Nr}$. 2, August 1989

[4] B. von Steiger, P. Baccini, Regionale Stolfbilanzierung von landwirtschuftlichen Böden mit messbarem Ein- und Austrag, Bericht Nr. 38 des Nationalen Forschungsprogramms 'Boden', 1990. 